\title{
Collective phenomena in heavy-ion collisions
}

\author{
Á Nyíri ${ }^{1}$, L P Csernai ${ }^{1,2}$, E Molnár ${ }^{1}$ and K Tamosiunas ${ }^{1}$ \\ ${ }^{1}$ Section for Theoretical and Computational Physics, University of Bergen, Allégaten 55, \\ N-5007 Bergen, Norway \\ 2 MTA-KFKI, Research Institute of Particle and Nuclear Physics, H-1525 Budapest, Hungary \\ E-mail: nyiri@ift.uib.no
}

Received 19 November 2004

Published 23 May 2005

Online at stacks.iop.org/JPhysG/31/S1045

\begin{abstract}
Collective flow from ultra-relativistic heavy-ion collisions is an important hadronic observable sensitive to the early stages of system evolution. We have calculated flow components from a tilted, ellipsoidally expanding source and investigated the dependence of flow pattern on the initial geometry of the fireball. We also pointed out possible problems connected to the experimental techniques used for the calculation of the $v_{n}$ Fourier coefficients, which may lead to serious inaccuracy in the flow analysis.
\end{abstract}

(Some figures in this article are in colour only in the electronic version)

\section{Introduction}

Collective flow was predicted to occur in relativistic heavy-ion collisions theoretically already in the early 1970s [1, 2] and proved to exist beyond doubt in 1984 first by the Plastic Ball Collaboration at LBL [3]. With time, a variety of collective flow patterns were detected. The increasing complexity of flow patterns naturally led to attempts to classify them in a more systematic way, on principal ground, and not just following the new experimental observations. This led to a formal classification of the azimuthal asymmetries via the coefficients, $v_{n}$, of the Fourier expansion of the azimuthal distribution of particles [4, 5]:

$$
E \frac{\mathrm{d}^{3} N}{\mathrm{~d}^{3} P}=\frac{1}{2 \pi} \frac{\mathrm{d}^{2} N}{p_{t} \mathrm{~d} p_{t} \mathrm{~d} y}\left(1+2 \sum_{n} v_{n}(y) \cos (n \Phi)\right),
$$

where $\Phi$ is the azimuth angle with respect to the true reaction plane of the event. In experiments anisotropic transverse flow manifests itself in the distribution of $\phi=\Phi+\Psi_{R}$, where $\phi$ is the measured azimuth for a track in detector coordinates, and $\Psi_{R}$ is the azimuth of the reaction plane in the event, which varies event by event in the coordinate frame of the detector. Using this definition the coefficients have a transparent meaning: $v_{n}=\left\langle\cos \left(n\left(\phi-\Psi_{R}\right)\right)\right\rangle$, where the average is taken over all emitted particles in a given rapidity, $y$, and transverse momentum, 
$p_{t}$, bin in all events. $\Psi_{R}$ cannot be directly measured, and randomly takes any value in $[0,2 \pi]$ due to the random direction of the impact parameter vector of the event.

Anisotropic flow has been fully recognized as an important observable providing information on the early stages of heavy-ion collisions [6-8]. The development of flow is closely related to the pressure gradients, and thus, to the equation of state of the nuclear matter formed in the collision [9, 10]. Collective flow is also believed to be a promising signal to detect the creation of the quark-gluon plasma [11-13]. An increased attention to collective flow has also resulted in significant improvements in the techniques and methods of analysis and presentation of the experimental data. PHENIX, PHOBOS and STAR experiments at RHIC produced a wealth of information on the flow components [14-21].

\section{Calculation of flow components}

We have calculated directed and elliptic flow from a tilted, ellipsoidally expanding particle emitting source. Our tool is a simple, blast-wave-type hydrodynamic model. The tilt angle, $\Theta$, represents the rotation of the major (longitudinal) direction of expansion from the direction of the beam. We have divided our fireball into cubic cells by a grid in $x, y, z$ coordinates, as it is done in most hydrodynamic models. The aim of the introduced discretization was to produce similar output as other models have, which makes it possible to change the presently used simple blast-wave model to more sophisticated ones without further changes in the next steps of the calculation.

\subsection{Theoretical background}

The contribution of a fluid cell to the final baryon phase-space distribution is [22]

$$
\frac{\mathrm{d} N_{c}}{\mathrm{~d}^{3} p}=\gamma V_{c} \frac{p^{\mu} \mathrm{d} \sigma_{\mu}}{p^{0}} f_{\text {F.O. }}(x, p),
$$

where $\gamma V_{c}$ is the proper volume of one fluid cell, $f_{\mathrm{F} . \mathrm{O}}(x, p)$ is the freeze-out distribution and $\mathrm{d} \sigma_{\mu}$ is the normal of the FO surface. Using the relations $p=\left(p^{0}, p_{\|}, \mathbf{p}_{\perp}\right), p_{\|}=p^{0} \mathrm{~d} y$ and $p_{t}=\left|\mathbf{p}_{\perp}\right|$, we can get the azimuthal distribution per unit rapidity as

$$
\begin{aligned}
\frac{\mathrm{d} N_{c}}{\mathrm{~d} y \mathrm{~d} \phi}=\int \frac{\mathrm{d}^{3} p}{\mathrm{~d} y \mathrm{~d} \phi} \frac{\mathrm{d} N_{c}}{\mathrm{~d}^{3} p}=\gamma V_{c} \int \frac{\mathrm{d}^{3} p}{\mathrm{~d} y \mathrm{~d} \phi} \frac{p^{\mu} \mathrm{d} \sigma_{\mu}}{p^{0}} f_{\mathrm{F} . \mathrm{O} .}(x, p) \\
=\gamma V_{c} \int \mathrm{d} p_{t} p_{t}\left(p^{\mu} \mathrm{d} \sigma_{\mu}\right) f_{\mathrm{F} . \mathrm{O} .}(x, p) \equiv \int \mathrm{d} p_{t} p_{t} G_{c}\left(p_{t}, \phi_{\mathrm{CM}}, y\right) .
\end{aligned}
$$

When we evaluate the azimuthal asymmetry this is done with respect to the reaction plane. The $\phi_{\mathrm{CM}}$ is the azimuth angle of particles in the centre of mass (CM) frame where these are measured. Then, the coefficients of the different harmonics, $v_{1}, v_{2}$, etc can be evaluated via additional numerical integrations over $\phi_{\mathrm{CM}}$ azimuth angle. Thus,

$$
v_{n}(y)=\frac{\sum_{c} \int \cos \left(n \phi_{\mathrm{CM}}\right) \gamma V_{c}\left(p^{\mu} \mathrm{d} \sigma_{\mu}\right) f_{\text {F.O. }}(x, p) \mathrm{d}^{2} p_{t}}{\sum_{c} \mathrm{~d} N_{c} / \mathrm{d} y} .
$$

\subsection{Results for directed and elliptic flow}

In this section we present a few results from our model calculations. The odd harmonics, like $v_{1}$, involve a forward/backward azimuthal antisymmetry. Therefore, without weighting 

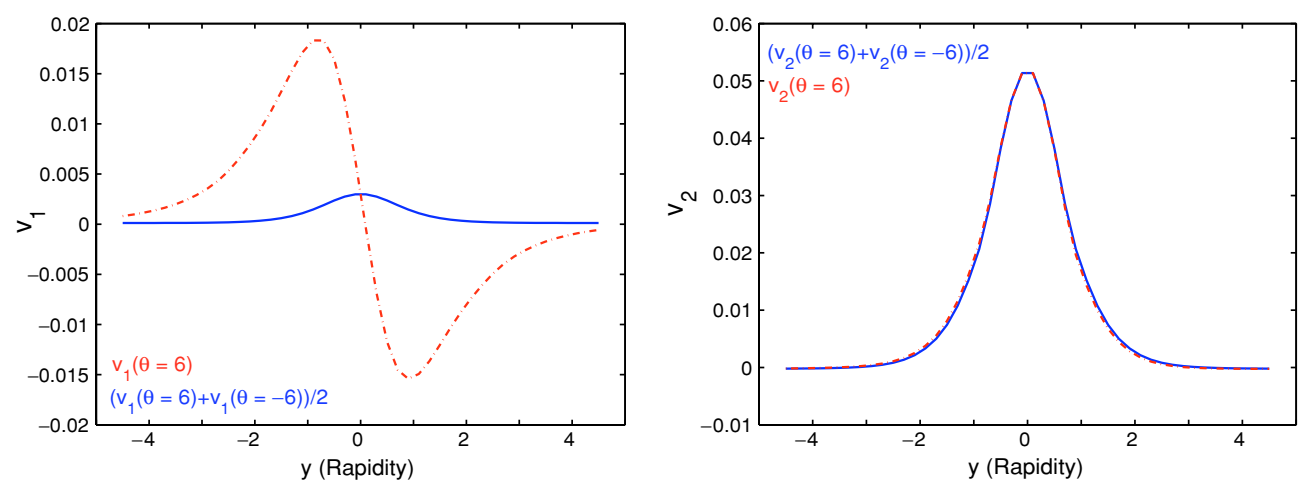

Figure 1. Left: directed flow, $v_{1}$, as a function of rapidity, $y$. Right: elliptic flow, $v_{2}$, as a function of rapidity, $y$. The chain curves refer to a source with tilt angle, $\Theta=6^{\circ}$, while the full curves are the average of flow components from two oppositely tilted ellipsoidal sources with tilt angles $\Theta=6^{\circ}$ and $\Theta=-6^{\circ}$. The half-axes of the ellipsoids were $a=10 \mathrm{fm}, b=8 \mathrm{fm}$ and $c=6 \mathrm{fm}$.

by rapidity the forward and backward contributions may cancel each other. To show this, we have calculated flow components from two tilted ellipsoidally expanding sources, which differ from one another only in the sign of the tilt angle.

The flow velocity in equation (4) is normal to the surface of the ellipsoid, and this way most cells have a flow velocity, which is orthogonal to the largest or most flat part of the surface, i.e. in the direction of the shortest half-axis, $c$, while the absolute value of the velocity was constant, $|v|=0.65 c$. As the ellipsoid is tilted for $b \neq 0$, this direction is not orthogonal to the beam axis, and results in azimuthal asymmetry, so, $v_{1} \neq 0$.

Our results are shown in figure 1 , where we have plotted the directed flow, $v_{1}$, and elliptic flow, $v_{2}$, as a function of rapidity, $y$. For even harmonics there is a symmetry for positive and negative $x$-values, thus the role of introducing weights with opposite signs for positive and negative rapidities in the event plane or cumulant method is not transparent. This may lead to the wrong conclusion that weighting is not important. The two results for $v_{2}$ are nearly identical, therefore one can hardly see that there are indeed two curves in the figure.

Figure 1 shows the results also for the directed flow, $v_{1}$. One can see, that $v_{1}(y)$ is definitely not constant zero and the so-called wiggle, which is well known from earlier experiments with lower energies, appears. The situation changes dramatically when we construct the 'averaged' $v_{1}$, which demonstrates what happens when we partly reverse the projectile and target side. As the full curve shows, in this case $v_{1}$ is in principle set to zero, $v_{1}(y) \approx 0$. The discretization to finite fluid cells leads to some inaccuracy in our calculations around mid-rapidity for small $\left(|\Theta|<10^{\circ}\right)$ flow angles.

We have also investigated the dependence of flow pattern on the initial geometry of the fireball. Such investigation might have an importance in understanding the initial stages of heavy-ion collisions. As the development of flow is related to the conditions in the nuclear matter formed in the collision, the measured flow might provide us with information also on the initial geometry if we know how the geometry affects the flow.

In figure 2, we have plotted the directed flow, $v_{1}$, and elliptic flow, $v_{2}$, as a function of rapidity, $y$, calculated assuming two ellipsoidal sources with the same thermodynamical properties but different geometries. Our results support the expectations derived from pure phenomenology that a highly deformed and tilted source develops stronger flow. However, recent experimental results on flow components do not support that the source can be so much 

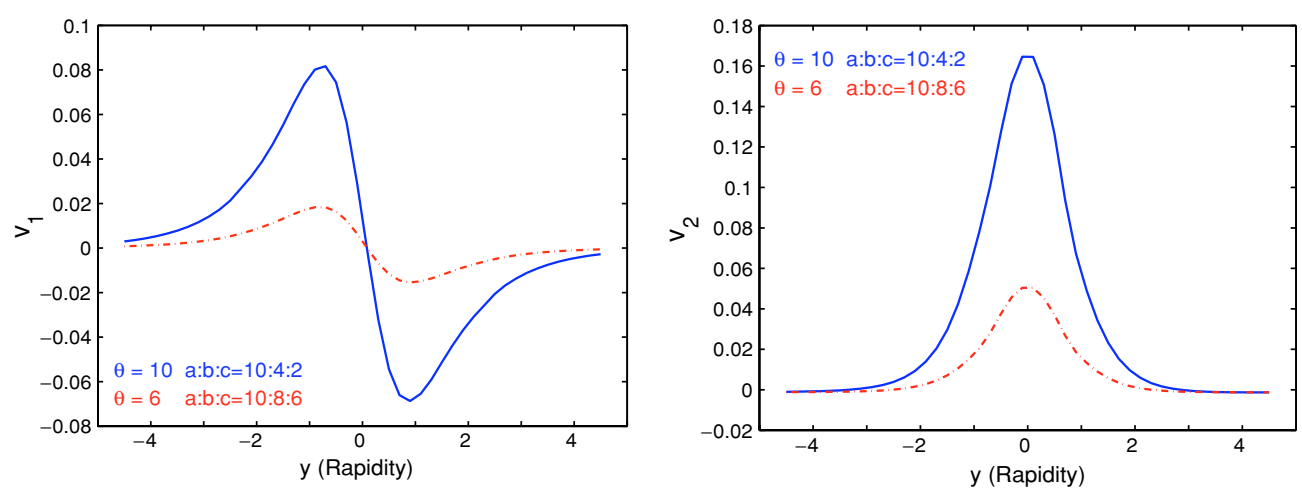

Figure 2. Left: directed flow, $v_{1}$, as a function of rapidity, $y$. Right: elliptic flow, $v_{2}$, as a function of rapidity, $y$. The chain curves refer to a source with tilt angle, $\Theta=6^{\circ}$, and half-axes $a=$ $10 \mathrm{fm}, b=8 \mathrm{fm}$ and $c=6 \mathrm{fm}$, while the full curves refer to a source with tilt angle, $\Theta=10^{\circ}$, and half-axes $a=10 \mathrm{fm}, b=4 \mathrm{fm}$ and $c=2 \mathrm{fm}$.

deformed as the measured flow components are much lower than those represented by full curves.

\section{Conclusions}

We have presented calculations of two flow components, the directed and elliptic flow. We found that model results for the first even harmonic, $v_{2}$, are in good agreement with the experimental data. However, the first odd harmonic, or directed flow, $v_{1}$, is very sensitive to the correct determination of the sign of the impact parameter and can be misinterpreted by some experimental methods. We have pointed out some critical points in the recently used experimental methods for calculation of flow components, which may lead to problems in the flow analysis. These arise from the possibility of misidentification of the reaction plane. Flow analysis is an important issue at RHIC, as it can provide us with valuable information on the early stages of the collisions, but it is not a trivial task. Therefore, further improvements of both the experimental techniques and theoretical models are needed. Especially, the reaction plane should be determined more accurately, since now the target and projectile sides may partly be reversed.

\section{Acknowledgments}

The authors wish to thank J Cleymans, R Adams and S Wheaton for their hospitality at the University of Cape Town, where parts of this work were done. ÁN acknowledges financial support from the Norwegian Research Council.

\section{References}

[1] Scheid W, Müller H and Greiner W 1974 Phys. Rev. Lett. 32741

[2] Chapline G F et al 1973 Phys. Rev. D 84302

[3] Gustafsson H A et al 1984 Phys. Rev. Lett. 53544

[4] Poskanzer A M and Voloshin S A 1998 Phys. Rev. C 58167

[5] Demoulins M et al 1990 Phys. Lett. B 241476

[6] Sorge H 1997 Phys. Rev. Lett. 782309 
[7] Zhang B, Gyulassy M and Ko C M 1999 Phys. Lett. B 45545

[8] Ollitrault J Y 1992 Phys. Rev. D 46229

[9] Reisdorf W and Ritter H G 1997 Ann. Rev. Nucl. Part. Sci. 47663

[10] Teaney D and Shuryak E V 1999 Phys. Rev. Lett. 834951

[11] Csernai L P and Röhrich D 1999 Phys. Lett. B 458454

[12] Brachmann J et al 2000 Phys. Rev. C 61024909

[13] Bleicher M and Stöcker H 2002 Phys. Lett. B 526309

[14] Adler C et al 2001 Phys. Rev. Lett. 87182301

[15] Adler C et al 2002 Phys. Rev. C 66034904

[16] Adler C et al 2003 Phys. Rev. Lett. 90032301

[17] Adams J et al 2004 Phys. Rev. Lett. 92062301

[18] Back B B et al 2002 Phys. Rev. Lett. 89222301

[19] Manly S et al 2003 Nucl. Phys. A 715611

[20] Adcox K et al 2002 Phys. Rev. Lett. 89212301

[21] Adler S S et al 2003 Phys. Rev. Lett. 91182301

[22] Csernai L P 1994 Introduction to Relativistic Heavy Ion Collisions (New York: Wiley) 